\title{
Association of gait speed and grip strength with risk of cardiovascular events in patients on haemodialysis: a prospective study
}

Atsumi Kuki ${ }^{1}$, Kentaro Tanaka ${ }^{2,3}$, Akifumi Kushiyama ${ }^{4 *}$ (D, Yoshihide Tanaka ${ }^{5}$, Shuta Motonishi ${ }^{6}$, Yasuji Sugano ${ }^{1}$, Toru Furuya ${ }^{7}$ and Takashi Ozawa ${ }^{8}$

\begin{abstract}
Background: Gait speed (GS) and handgrip strength (HGS), both factors associated with frailty and sarcopenia, are reportedly associated with CV events in the general population. However, little is known about the impact of these factors on the outcome of patients on dialysis. This study aimed to evaluate whether evaluation of GS and HGS could be associated the onset of fatal/non-fatal cardiovascular (CV) events in patients on haemodialysis (HD).

Methods: One-hundred-eighty-two patients with end-stage renal disease (ESRD) undergoing HD at four dialysis clinics in April 2015 provided written informed consent to participate in the study. We excluded patients who had physical disability, were unable to walk without help, or had recently experienced CV events. Usual GS over a 4-m walk and HGS were measured at baseline, and 173 patients (men, 124; women, 49) were divided into sex-specific quartiles according to GS and HGS and were followed-up for fatal/non-fatal CV events for a median of 2 years. We examined the association of GS and HGS with CV events and determined cut-off values using Cox regression analysis adjusted for age, sex, HD duration, history of CVD, and diabetes.

Results: During the follow-up period, $46 \mathrm{CV}$ events occurred. Both physical performance factors were significantly associated with CV events. Low GS ( $<0.82 \mathrm{~m} / \mathrm{s}$ for men and $0.81 \mathrm{~m} / \mathrm{s}$ for women) and weak HGS $(<29.0 \mathrm{~kg}$ for men and $19.7 \mathrm{~kg}$ for women) were associated with CV events. For low vs. high GS, the hazard ratio (HR) for CV events was 2.29 [95\% confidence interval $(\mathrm{Cl}): 1.20-4.33 ; P=0.01]$, and for low vs. high $\mathrm{HGS}$, the HR was 2.15 [95\% Cl: 1.00-5.04; $P<0.05]$. These HRs remained significant after adjusting for confounding factors, such as sex, age, dialysis vintage, history of CV disease, and diabetes.
\end{abstract}

Conclusions: Slow GS and weak HGS in patients on HD were suggested to be independent predictors of fatal/nonfatal CV events.

Keywords: Chronic haemodialysis, Physical activity, Prognosis, Cardiovascular events

\footnotetext{
* Correspondence: kusiyaa-tky@umin.ac.jp

${ }^{4}$ Department of Pharmacotherapy, Meiji Pharmaceutical University, 2-522-1

Noshio, Kiyose, Tokyo 204-8588, Japan

Full list of author information is available at the end of the article
}

(c) The Author(s). 2019 Open Access This article is distributed under the terms of the Creative Commons Attribution 4.0 International License (http://creativecommons.org/licenses/by/4.0/), which permits unrestricted use, distribution, and reproduction in any medium, provided you give appropriate credit to the original author(s) and the source, provide a link to the Creative Commons license, and indicate if changes were made. The Creative Commons Public Domain Dedication waiver (http://creativecommons.org/publicdomain/zero/1.0/) applies to the data made available in this article, unless otherwise stated. 


\section{Background}

With the increase in the ageing Japanese population, there is an increase in the number of aging patients with end stage renal disease (ESRD) [1]. The average age of Japanese dialysis patients was 51.3 years in 1983, which reached 69.4 years by the end of 2016 [1]. Frailty and sarcopenia are highly prevalent in elderly patients with ESRD undergoing dialysis [2-4]. The deterioration of renal function leads to a variety of metabolic disorders in patients with ESRD, including chronic inflammation, uraemia, oxidative stress, insulin resistance, and malnutrition, which increases the risk of frailty and sarcopenia $[5,6]$.

Frailty and sarcopenia were associated with poor outcomes, including not only falls, incident disability, hospitalization, and mortality $[7,8]$, but also cardiovascular (CV) events [9], such as myocardial infarction (MI), heart failure, and cerebrovascular disorders. The frailty assessment includes the following five items in the frailty phenotype protocol: weight loss, exhaustion, low physical activity level, low handgrip strength (HGS) as weakness, and slow gait speed (GS) as physical disability [10]. Sarcopenia is mainly characterized by atrophy of skeletal muscles, along with a decrease in muscle strength and function and exhibits physical function impairment-a common aspect of frailty [11]. Previous studies have suggested that several features of frailty or sarcopenia, such as exhaustion, slow GS, low HGS, low physical activity level, and low skeletal muscle mass, constitute the criteria associated with the onset of $\mathrm{CV}$ events [12, 13].

GS and HGS are the common physical parameters associated with frailty and sarcopenia. These two parameters have been reported to be potential prognostic indicators of $\mathrm{CV}$ events in the general elderly population [12]. According to a previous systematic review, GS is significantly associated with cardiovascular risk factors (hypertension, diabetes, and intima-media thickness) and $\mathrm{CV}$ events (peripheral artery disease [PAD], stroke, and CV mortality) in the older population [14]. HGS is a well-established indicator of overall muscle strength. A previous meta-analysis reported a linear association between HGS and CV events in a community-dwelling population [15]. Furthermore, HGS was found to be inversely associated with $\mathrm{CV}$ events in a study including 1.1 million young Swedish men [16].

In patients undergoing $\mathrm{HD}, \mathrm{CV}$ events are the leading cause of death [17], however, little information is available with regards to quantitative risk assessment for $\mathrm{CV}$ events by frailty and sarcopenia. Therefore, this study aimed to prospectively evaluate whether the two physical performance parameters are associated with the onset of fatal/non-fatal CV events in patients undergoing HD. In particular, we also examined whether there were thresholds in the two measurements., with certain threshold.

\section{Methods}

\section{Trial design}

The present study was a 2-year prospective observational cohort study to examine the association between GS/HGS and CV events in patients with ESRD undergoing $\mathrm{HD}$ at multiple clinics. The protocol was approved by the Institutional Ethics Committee of Medical Toyou, Japan, and we obtained written informed consent from all participants after the protocol was explained in detail. This study was conducted in accordance with the ethical standards of the responsible committee on human experimentation and with the principles of the Helsinki Declaration.

\section{Study population}

We recruited 182 outpatients with chronic kidney disease (CKD) stage-5 undergoing HD at our four outpatient dialysis clinics (Kitahachiouji Clinic, Higasikurume Clinic, Higashiyamato Nangai Clinic, and Kodairakitaguchi Clinic) in Tokyo, Japan, from January 2015 to March 2015.

Eligibility criteria included age $\geq 20$ years at enrolment and undergoing HD three times per week for more than 3 months. Patients were excluded if they exhibited physical disability (Barthel index <90) [18] or had a history of $\mathrm{CV}$ events within 1 month before the measurement of GS and HGS. We excluded nine patients with physical disability $(n=7)$, and with a recent history of CV events $(n=2)$. The remaining 173 patients $(124$ men and 49 women, age 34-91 years) were enrolled in the study (Fig. 1).

\section{Data collection}

Demographic and clinical data, including age, sex, and comorbidities were collected by retrieving medical records and asking the patients directly. Laboratory parameters and dialysis adequacy were measured before the first HD session of the week. The single-pool Kt/V was used to determine the weekly dialysis dose. Subjects with diabetes were defined as those who had a previous diagnosis of diabetes by a physician or had a haemoglobin A1c (HbA1c) level > 6.5\%. History of CVD included history of medical therapy or surgical treatment for MI, angina, PAD, and hospitalization for congestive heart failure or stroke.

\section{Physical performance parameters}

Physical performance was assessed at the baseline. A walking test was performed along a 4-m walkway with a 1-m start-up from the starting point at individual patients' usual pace. All participants started to walk from the standing position. GS $(\mathrm{m} / \mathrm{s})$ was calculated by dividing the distance covered by time (s) [19]. 


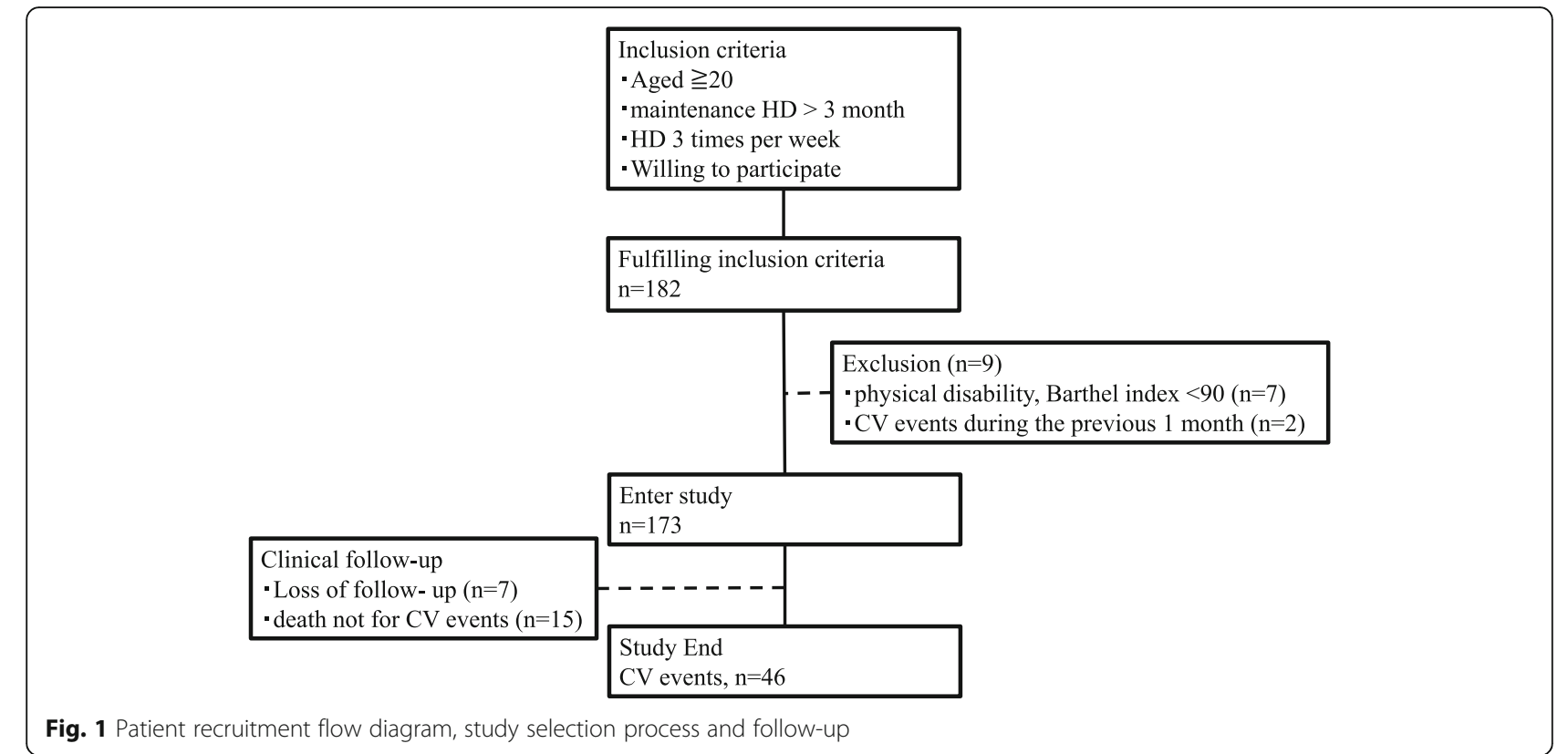

HGS was measured using a hand-held dynamometer (Takei Scientific Instruments Co, Niigata, Japan). After adjusting the dynamometer for hand comfort, the HGS (in $\mathrm{kg}$ ) was measured in a sitting position with the arm hanging by the side and the elbow fully extended. The highest grip strength after maximal efforts was recorded for each hand twice. For the present analysis, we used the maximum values obtained from both hands. Both usual gait speed and grip force measurements were performed before the dialysis session on either the first, second, or third dialysis day of the week.

\section{Ascertainment of cardiovascular events}

The primary endpoint was incident $\mathrm{CV}$ events during the observational period from 1 April 2015 to 1 April 2017. Patients were reviewed during a median 2-year follow-up period by medical records and information from interviews. Follow-up was performed from enrolment until the first event, or loss to follow-up due to any reason, such as transfer to another clinic, whichever occurred first. CV events were defined as CV death, MI, stroke, angina, hospitalizations for congestive heart failure, and PAD.

MI and angina were confirmed using coronary angiography or myocardial scintigraphy. Stroke was defined as haemorrhagic or ischaemic stroke, which included lacunar infarctions with symptoms confirmed by brain computed tomography $(\mathrm{CT})$ or magnetic resonance imaging (MRI). PAD was diagnosed using angiography with enhanced CT or MRI. Only one first event per subject was included in the analysis. The onset of $\mathrm{CV}$ events without sudden death was defined as the medical or surgical treatment initiation date.

\section{Statistical analysis}

Subjects were divided into sex-specific quartiles, ranging from Q1 (the lowest quartile) to Q4 (the highest quartile), according to baseline GS and HGS. The cut-offs were as follows: GS $(\mathrm{m} / \mathrm{s}):<0.82,0.82-0.95,0.95-1.15$, and $>1.15$ for men, and $<0.81,0.81-0.93,0.93-1.11$, and $>1.11$ for women; HGS $(\mathrm{kg})$ : $<22.1,22.1-26.2$, $26.2-32.1$, and $>32.1$ for men, and $<15.0,15.0-17.1$, $17.1-21.6$, and $>21.6$ for women.

Demographic data were summarized using the mean and standard deviation or median with 25th and 75th percentiles for continuous variables, as appropriate; and count and proportion for categorical data. Spearman's correlation analysis was used to analyse the association between GS and HGS. Comparison of baseline characteristics between the GS and HGS quartiles was performed using the Kruskal-Wallis test and CochranArmitage trend test for continuous and categorical variables, respectively. The incidence rates of $\mathrm{CV}$ events were estimated via the Kaplan-Meyer method and compared using a log-rank test according to GS and HGS categories.

Cox proportional hazards models were used to evaluate the association between GS and HGS and CV events. To evaluate the threshold, GS and HGS were divided into 16 sex-specific quantiles. At each quantile point (6.25th percentile), we made two groups-below and above the quantile-and calculated the hazard ratios (HRs) and 95\% confidence intervals (CIs) for the low vs. high GS and HGS groups. Multivariate Cox regression analyses were performed to determine independent prognostic effect of GS and HGS, divided according to the obtained threshold values for $\mathrm{CV}$ events, after 


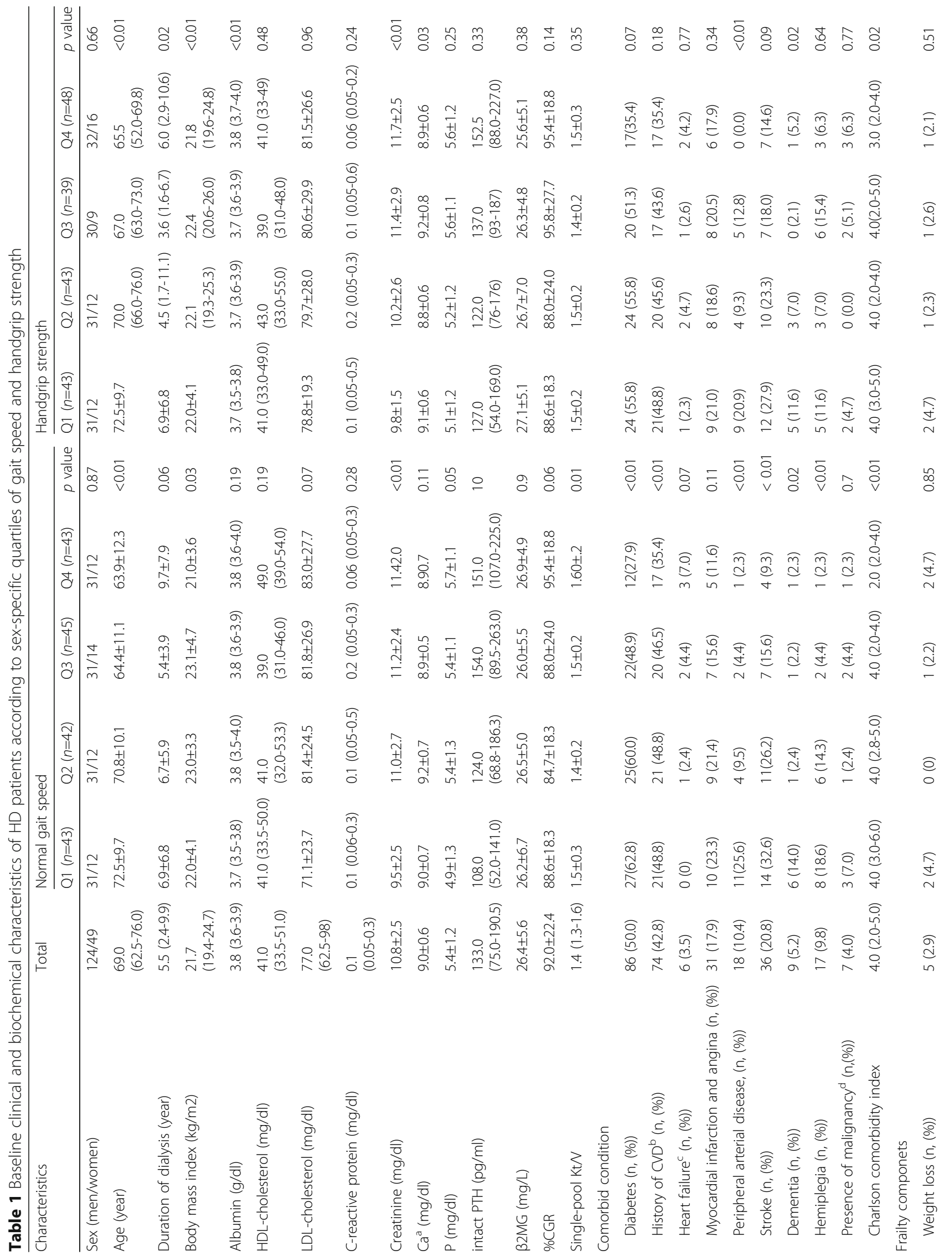




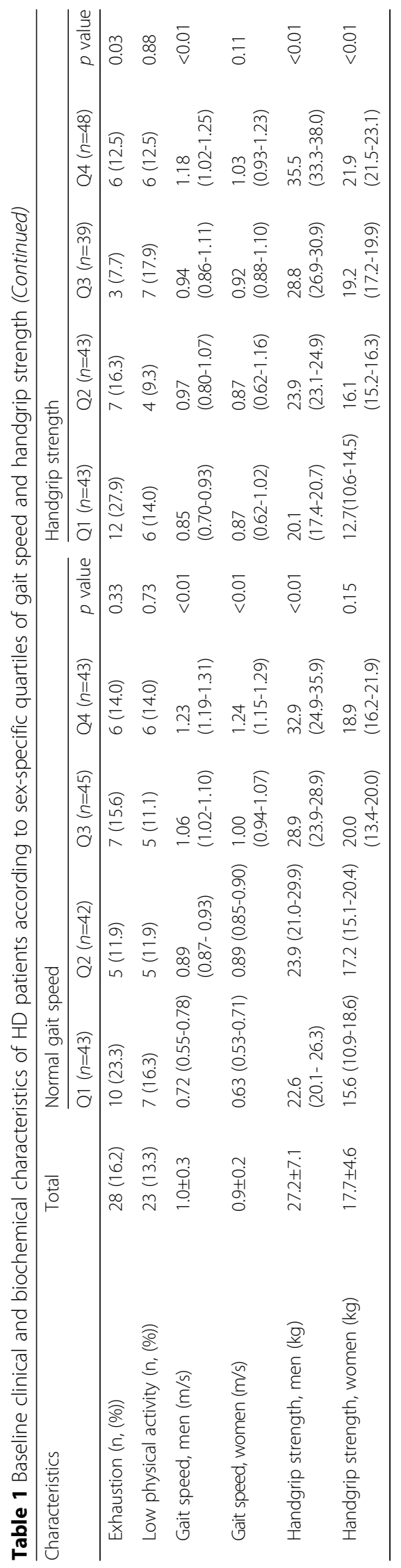



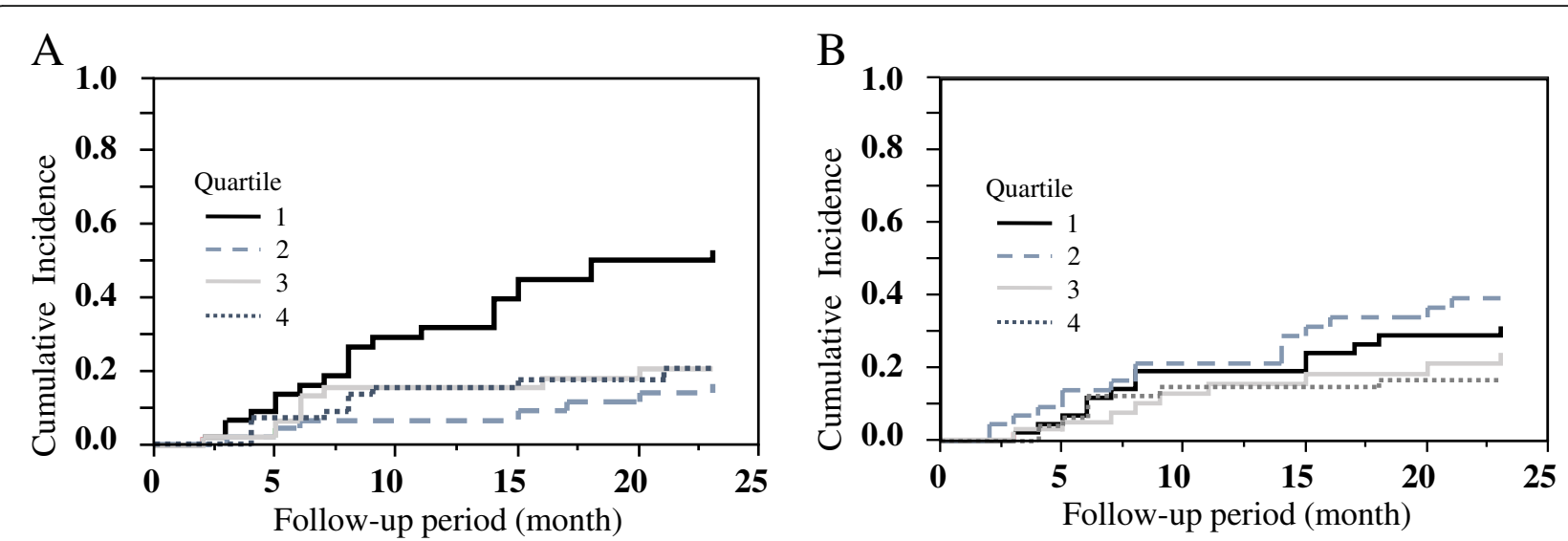

Number at risk

$\begin{array}{llllllllllllll}\text { Q1 } & 43 & 39 & 27 & 23 & 19 & 18 & \mathrm{Q} 1 & 43 & 41 & 35 & 33 & 29 & 28 \\ \text { Q2 } & 42 & 41 & 40 & 39 & 36 & 35 & \text { Q2 } & 43 & 39 & 34 & 28 & 25 & 23 \\ \text { Q3 } & 45 & 44 & 36 & 36 & 32 & 31 & \text { Q3 } & 39 & 38 & 34 & 31 & 28 & 27 \\ \text { Q4 } & 43 & 39 & 37 & 36 & 34 & 33 & \text { Q4 } & 48 & 46 & 42 & 42 & 40 & 39\end{array}$

Fig. 2 Cumulative incidence of cardiovascular events based on sex-specific quartiles of GS (a) and HGS (b). The sex-specific cut-offs for GS were $<0.82 \mathrm{~m} / \mathrm{s}, 0.82-0.95 \mathrm{~m} / \mathrm{s}, 0.95-1.15 \mathrm{~m} / \mathrm{s}$, and $>1.15 \mathrm{~m} / \mathrm{s}$ in men, and $<0.81 \mathrm{~m} / \mathrm{s}, 0.81-0.93 \mathrm{~m} / \mathrm{s}, 0.93-1.11 \mathrm{~m} / \mathrm{s}$, and $>1.11 \mathrm{~m} / \mathrm{s}$ in women. The sex-specific cut-offs for HGS were $<22.1 \mathrm{~kg}, 22.1-26.2 \mathrm{~kg}, 26.2-32.1 \mathrm{~kg}$, and $>32.1 \mathrm{~kg}$ in men, and $<15.0 \mathrm{~kg}, 15.0-17.1 \mathrm{~kg}, 17.1-21.6 \mathrm{~kg}$, and $>21.6 \mathrm{~kg}$ in women

adjusting for the following confounders: age, sex, HD duration, history of CVD, and diabetes. All analyses were performed using JMP software (version 13.0; SAS Institute, Cary, NC, USA). Statistical significance was set at $P<0.05$.

\section{Results}

In the present study, 173 patients with ESRD (124 men and 49 women) undergoing HD were enrolled. Table 1 shows the baseline clinical characteristics of all patients and subgroups according to GS and HGS quartiles. The median (interquartile-range [IQR]) age was 69.0 years (62.5-76.0 years), and $71.7 \%$ of participants were male. The patients' median (IQR) HD duration was 5.5 years (2.4-9.9 years). Fifty percent of the patients were diabetic, and $42.8 \%$ had a history of CVD. The mean GS was $1.0 \pm 0.3 \mathrm{~m} / \mathrm{s}$ for men and $0.9 \pm 0.2 \mathrm{~m} / \mathrm{s}$ for women, and the mean HGS was $27.2 \pm 7.1 \mathrm{~kg}$ for men and $17.7 \pm$ $4.6 \mathrm{~kg}$ for women. GS correlated with HGS in both men $(\rho=0.49, P<0.01)$ and women $(\rho=0.36, P=0.03)$ by Spearman's correlation analysis.

We observed a significant GS quartile-dependent difference in age $(\mathrm{P}<0.01)$, body mass index $(\mathrm{BMI})$ $(P=0.03)$, serum creatinine level $(P<0.01)$, single-pool Kt/V $(P=0.01)$, dementia $(P=0.02)$, Charlson comorbidity index $(\mathrm{CCI})(P<0.01)$ [20], the presence of diabetes $(P<0.01)$, history of CVD $(P<0.01)$, and hemiplegia $(P<0.01)$. HGS showed a significant quartile-dependent difference in age $(P<0.01)$, BMI $(P=0.02)$, serum albumin $(P<0.01)$, creatinine $(P<0.01)$, calcium $(P=0.03)$, the presence of dementia $(P=0.02)$, CCI $(P=0.02)$, exhaustion $(P=0.03)$. The presence of diabetes and a history of CVD showed no significant difference across the four HGS quartiles.

During the 2-year follow-up period, CV events occurred in 46 patients $(26.6 \%)$ : five (3.0\%) had CV-related death, 12 (6.9\%) experienced MI and angina, 11 (6.4\%) experienced stroke, eight (4.6\%) were hospitalized for congestive heart failure, and ten (5.8\%) experienced PAD. The incidence rate of total CV events was 17.0 per 100 person-years at risk. The incidence rates of $\mathrm{CV}$ death, MI, MI and angina, stroke, congestive heart failure, and PAD were 1.7, 1.0, 5.0, 4.4, 3.4, and 4.7 per 100 person-years. The rates of MI and angina were the highest among the $\mathrm{CV}$ events in $\mathrm{HD}$ patients. Analysis according to the quartiles of GS revealed that the 2-year cumulative incidence rates were 53.1, 16.8, 20.8, and 21.0 for Q1 through to Q4, respectively. There was a significant difference in $\mathrm{CV}$ events across the stratified GS. Patients in the lowest quartile had a significantly higher risk of $\mathrm{CV}$ events than those in the higher three quartiles (log-rank test, $P=0.0005$ ) (Fig. 2a). The 2-year cumulative incidence rates were $31.5,39.3,24.2$, and 16.7 for Q1 through Q4 for HGS. There was no significant difference in CV events across various HGSs (Fig. 2b).

In the univariate Cox regression analysis, old age, diabetes, history of CVD, low creatinine generation rate, low GS, and low HGS were revealed to be significantly associated with $\mathrm{CV}$ events when these explanatory variables were treated as continuous variables (Table 2). 
Table 2 Clinical and biochemical parameters affecting CV events by univariate Cox model

\begin{tabular}{lll}
\hline Factors & Hazard ratio $(95 \% \mathrm{Cl})$ & $p$ value \\
\hline Age (years) & $1.04(1.01-1.07)$ & 0.01 \\
Gender (M) & $0.89(0.48-1.71)$ & 0.71 \\
Dialysis duration (years) & $0.98(0.93-1.02)$ & 0.39 \\
Diabetes & $1.87(1.04-3.47)$ & 0.04 \\
History of CVD & $2.34(1.31-4.31)$ & $<0.01$ \\
Charlson comorbidity index & $1.24(1.04-1.46)$ & 0.02 \\
\%CGR & $0.98(0.97-0.99)$ & 0.04 \\
BMI (kg/m2) & $1.02(0.95-1.10)$ & 0.55 \\
GNRI & $1.06(0.51-2.01)$ & 0.87 \\
Haemoglobin (g/dL) & $1.27(0.92-1.73)$ & 0.14 \\
Albumin (g/dL) & $0.47(0.16-1.31)$ & 0.15 \\
LDL-cholesterol (mg/dL) & $1.01(0.99-1.02)$ & 0.36 \\
intact PTH (pg/mL) & $1.00(1.00-1.00)$ & 0.63 \\
C-reactive protein (mg/dL) & $1.24(0.85-1.60)$ & 0.22 \\
Gait speed (m/s) & $0.16(0.05-0.54)$ & $<0.01$ \\
Handgrip strength (kg) & $0.96(0.92-0.99)$ & 0.04 \\
\hline CVD cardovasclar disease $\% C G R \%$
\end{tabular}

CVD cardiovascular disease, \%CGR \% creatinine generation rate,

$B M I$ body mass index, GNRI geriatric nutritional risk index

${ }^{\mathrm{a}} \mathrm{GNRI}=14.89 \times$ albumin $(\mathrm{g} / \mathrm{dL})+41.7 \times($ body weight/ideal body weight $)$

To explore the thresholds of GS and HGS for CV events, patients were classified into two groups, above and below each quantile of GS and HGS, which were further divided into 16 quantiles (Fig. 3). The unadjusted HRs for GS rose from the middle of Q1 (12.5th percentile) and became highest (HR: 3.25 , 95\% CI: $1.22-$ $2.67 ; P<0.01)$ at $0.82 \mathrm{~m} / \mathrm{s}$ in men and $0.81 \mathrm{~m} / \mathrm{s}$ in women between Q1 and Q2 (25th percentile) (Fig. 3a). The unadjusted HRs of HGS peaked at the middle of Q3 (62.5th percentile) (HR: $3.76,95 \%$ CI: $1.16-23.0 ; P=0.02$, at $29.0 \mathrm{~kg}$ in men and $19.7 \mathrm{~kg}$ in women) and the second peak was seen in the middle of Q4 (87.5th percentile) (HR: 3.76, 95\% CI: $1.16-23.0 ; \mathrm{P}=0.02$, at $35.7 \mathrm{~kg}$ in men and $22.1 \mathrm{~kg}$ in women) (Fig. 3c). After adjustment for age, sex, dialysis vintage, diabetes, and history of CVD, both GS and HGS were found to be independently associated with CV events. Participants in the <25th percentile of GS (Fig. 3b), and those in the $<62.5$ th percentile of HGS (Fig. 3d) exhibited a HR of 2.29 (95\% CI: 1.20-4.33; $P=0.01$ ), and 2.15 (95\% CI: $1.00-5.04 ; P<0.05)$ for $\mathrm{CV}$ events compared with the remaining participants. These thresholding quantile points of unadjusted and adjusted HRs were consistent; the GS threshold was $0.82 \mathrm{~m} / \mathrm{s}$ in men and $0.81 \mathrm{~m} / \mathrm{s}$ in women, while that of HGS was $29.0 \mathrm{~kg}$ in men and $19.7 \mathrm{~kg}$ in women.

\section{Discussion}

In this study, among Japanese HD outpatients who were able to walk independently, slow usual GS and weak HGS were significantly associated with $\mathrm{CV}$ events, independent of age, sex, HD duration, and medical history. When referring to the incidence rate of $\mathrm{CV}$ events between $\mathrm{HD}$ and non-HD patients in the previous studies (Additional file 1: Table S1), the incidence rate of MI and stroke in HD patients are higher than those of the general population.

The cut-off points of GS were identified as $0.82 \mathrm{~m} / \mathrm{s}$ in men and $0.81 \mathrm{~m} / \mathrm{s}$ in women, and those of HGS were $29.0 \mathrm{~kg}$ in men and $19.7 \mathrm{~kg}$ in women; values below these cut-offs indicated a significant association with CV events. The proportion of patients below the cut-off for GS was small, while that of patients below the cut-off for HGS was more than half. This is the first study to indicate the cut-off points of usual GS and HGS for CV events in patients with ESRD undergoing HD.

The threshold of GS for CV events could vary owing to subjects' background and studies' endpoints. Fatal CV was associated with $\mathrm{GS}<0.8 \mathrm{~m} / \mathrm{s}$ at a mean age of 76 years in Iceland [21], or $<1.3 \mathrm{~m} / \mathrm{s}$ for men and $<1.1 \mathrm{~m} / \mathrm{s}$ for women in general civil servants in London [22]. The Iceland cohort were aged average 76 and $6 \mathrm{~m}$ walk test whereas those in London were 61 i.e. more than a decade younger and $2.44 \mathrm{~m}$ walk test. The threshold of GS has been revealed to be < $0.9 \mathrm{~m} / \mathrm{s}$ to affect the incidence of PAD in several studies [23-25]. In addition, a GS of $<0.8 \mathrm{~m} / \mathrm{s}$ has been associated with ischaemic heart disease, heart failure, and stroke in a cross-sectional study $[26,27]$. Variation in the GS threshold across previous studies might be due to the diversity of protocols, walking distances, measurement methods, and starting positions [28]. Walking distances ranged from $2.44-20 \mathrm{~m}$, although the 4-m assessment has been frequently reported in the literature [14]. The cut-off points of GS in the study are similar to the cut-off points $0.8 \mathrm{~m} / \mathrm{s}$ for defining low physical performance in sarcopenia [29].

HGS is a strong predictor of CVD. In community-dwelling populations, the multivariate-adjusted $\mathrm{HR}$ for $\mathrm{CV}$ events per $5 \mathrm{~kg}$ decreases in HGS was 1.21 (1.14-1.29, $\mathrm{I}^{2}=$ 95.5\%); a linear association between HGS within $56 \mathrm{~kg}$ and $\mathrm{CV}$ events was reported to be significant [15]. For specific $\mathrm{CV}$ events, the multivariate-adjusted $\mathrm{HR}$ associated with a $5 \mathrm{~kg}$ HGS decrease for coronary heart disease $\left(1.07,1.03-1.11, \mathrm{I}^{2}=91.6 \%\right)$ and stroke $\left(1.09,1.05-1.14, \mathrm{I}^{2}=91.0 \%\right)$ was found to be significant. Several studies have also shown that HGS is significantly associated with CV-related death in both men and women [30-32], and with heart failure, stroke, and ischemic heart disease in men [16]. The thresholds for $\mathrm{CV}$-related death were $<28 \mathrm{~kg}$ in men and $<17 \mathrm{~kg}$ in women aged $>65$ years, $<47.0 \mathrm{~kg}$ in men and $<24.0 \mathrm{~kg}$ in women aged $40-64$ years [33], and $<30.0 \mathrm{~kg}$ in Japanese men and $<16.5$ in Japanese women aged $>65$ years [32]. In HD patients, thresholds 


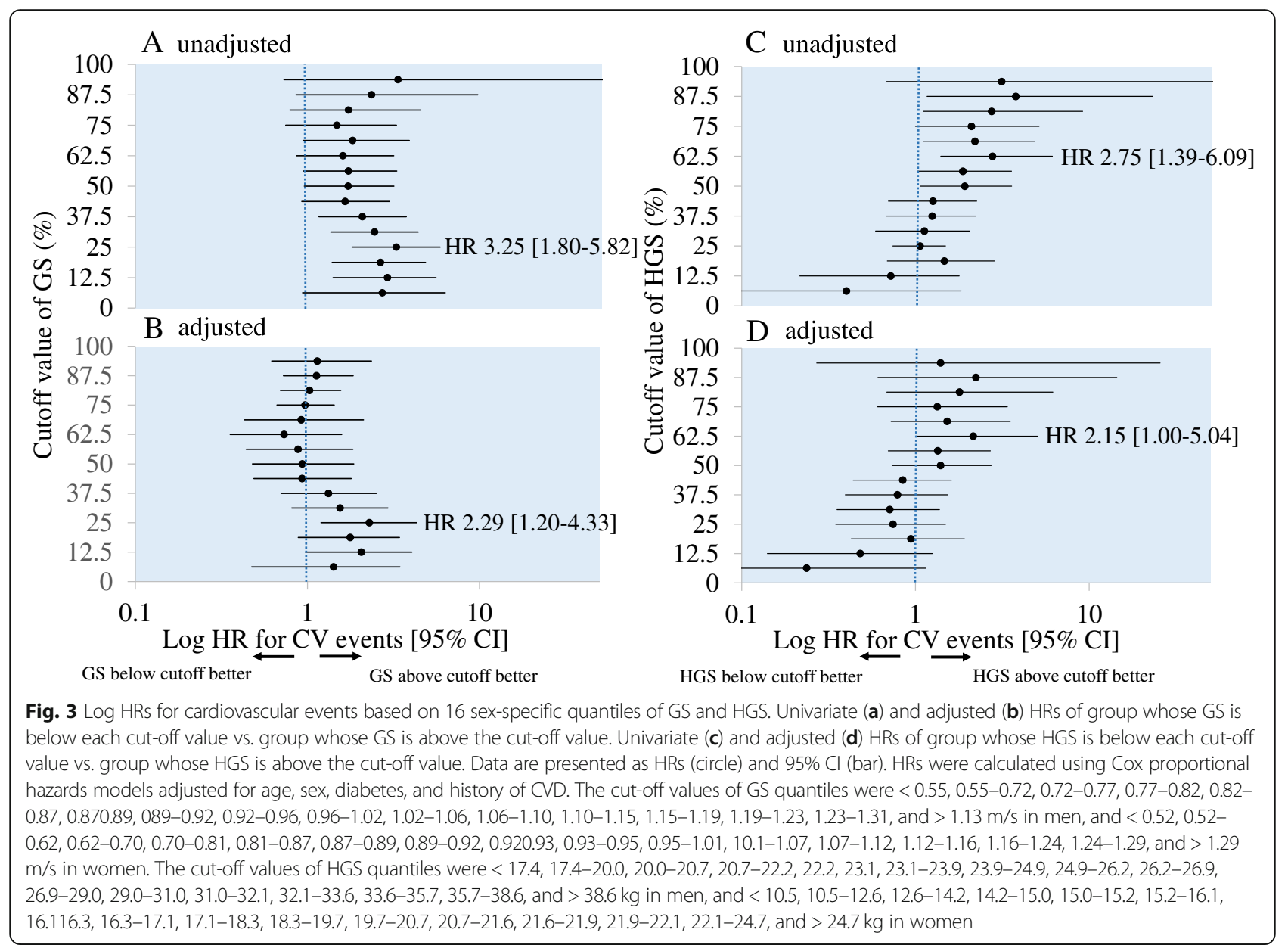

for such CV events have been reported to be $<30.0 \mathrm{~kg}$ in men and $<20.0 \mathrm{~kg}$ in women [34], similar to the results observed in the present study. However, protocols for HGS evaluation among studies were not consistent with respect to the arm position of measurement, choice of arm side, the reference to use [30, 32-35], and in the standing or seated position [36, 37]. In addition, fistula placement in HD may influence the HGS. HGS was reported to be significantly lower in the access-side limb than in the opposite side after arteriovenous fistula placement [38], and was directly related to thenar oxygenation and to finger systolic pressure only in the access-side extremity [39]. There is a need for the standardization of the techniques used for HGS evaluation in HD patients.

The mechanisms to explain the association of GS and HGS with CV events are not fully understood. For GS, walking was the most common and accessible mode of physical activity, and physical activity partially prevents age-related cardiac remodelling [40]. Physical activity has beneficial effects on obesity, diabetes, lipid profile, endothelial function and insulin sensitivity, and inflammation, which are all important risk factors of CV events [41]. A recent British cohort suggested that increased relative exercise intensity provides a greater stimulus for physiological adaptations known to influence CV-related mortality [41]. Similarly, the effect of low muscle strength is mediated through a reduced incidence of abdominal adiposity, weight gain, insulin resistance, metabolic syndrome, hypertension, and chronic inflammation [11, 12, 42]. A recent review reported that HGS could be an overall indicator of the integrity of the central nervous system and could reflect changes in the aging process [43].

The current study has some limitations. First, a common limitation of observational studies is that other unmeasured confounding factors of $\mathrm{CV}$ events are of concern. HD patients have CKD-related CV risk factors as well as traditional risk factors. We did not evaluate several CKD-related CV risks such as overload/ultrafiltration volumes, oxygen saturation and medicines e.g. opioids and sedatives. Second, this study was conducted only for the Japanese population in Japan, and there are possibilities for the different degrees of impact of GS and HGS from other ethnicities or other circumstances of HD. However, aging of HD patients and increasing frailty and sarcopenia is expected to become a worldwide issue $[2,4,44]$. 
Finally, more interventional studies on physical performance measures should be performed to elucidate whether an improvement in GS and/or HGS above the thresholds could reduce the incidence of $\mathrm{CV}$ events.

\section{Conclusions}

We conclude that poor walking performance (men $<0.82$ $\mathrm{m} / \mathrm{s}$, women $<0.81 \mathrm{~m} / \mathrm{s}$ ) and weak HGS (men $<29.0 \mathrm{~kg}$, women $<19.7 \mathrm{~kg}$ ) are suggested to be increased CV events in HD patients without physical disability. The walking test and grip force test are inexpensive and relatively simple to measure for the cardiovascular assessment of HD patients in daily practice.

\section{Additional file}

Additional file 1: Table S1. Comparison of the incidence of cardiovascular disease among cohorts in the United States and Japan. (DOCX $15 \mathrm{~kb}$ )

\section{Abbreviations \\ BMI: Body mass index; CCl: Charlson comorbidity index; Cl: Confidence interval; CKD: Chronic kidney disease; CT: Computed tomography; CV: Cardiovascular; CVD: Cardiovascular disease; ESRD: End-stage renal disease; GS: Gait speed; HbA1c: Haemoglobin A1c; HD: Haemodialysis; HGS: Handgrip strength; HR: Hazard ratio; MI: Myocardial infraction; MRI: Magnetic response imaging; PAD: Peripheral artery disease}

\section{Acknowledgments}

The authors wish to thank all the patients who participated in this study. The authors also thank Yui Izumi, Nao Okumura, and Yukiko Uchiyama for the physical performance evaluation of the patients.

\section{Funding}

The authors have no relevant affiliations and financial involvement with any organization.

\section{Availability of data and materials}

Data sets generated and/or analysed during the current study are not publicly available because they are part of the medical records of the patients involved but could be made available by the corresponding author upon reasonable request.

\section{Authors' contributions}

AK (Atsumi Kuki) designed the study and wrote the manuscript. KT contributed to the selection of objects, collection and analysis of data, and preparation of articles. AK (Akifumi Kushiyama) contributed to the conception and design of the study, interpretation of data, and made revision of manuscript for key intellectual content. YT, SM, YS, TF and TO collected data, and prepared articles. All the authors have read the manuscript and have approved its submission.

\section{Ethics approval and consent to participate}

The study protocol was approved by the Institutional Ethics Committee at Medical Toyou, Japan. Permission to conduct the study was obtained from each site based on the approval. Each patient had provided their signed written informed consent before they participated in this study.

\section{Consent for publication}

Not applicable.

\section{Competing interests}

The authors declare that they have no competing interests.

\section{Publisher's Note}

Springer Nature remains neutral with regard to jurisdictional claims in published maps and institutional affiliations.

\section{Author details}

${ }^{1}$ Kitahachiouji Clinic, 2960-5 Ishikawa-cho, Hochiuji-shi, Tokyo 1920032, Japan. ${ }^{2}$ Higashikurume Ekimae Clinic, 1-3-6 Honmachi, Higashikuruume-shi, Tokyo 2030053, Japan. ${ }^{3}$ The Division of Diabetes and Metabolism, The Institute for Adult Diseases, Asahi Life Foundation, 2-2-6, Bakuro-cho, Chuo-ku, Tokyo 103-0002, Japan. ${ }^{4}$ Department of Pharmacotherapy, Meiji Pharmaceutical University, 2-522-1 Noshio, Kiyose, Tokyo 204-8588, Japan. ${ }^{5}$ Kumegawa tousekinaika Clinic, 3-6-3 Onda-cho, Musashimurayama-shi, Tokyo 189-0011, Japan. ${ }^{6}$ Higashiyamato Nangai Clinic, 4-2-8 Nangai, Higashiyamato-shi, Tokyo 2070014, Japan. ${ }^{7}$ Higashikurume Clinic, 2-2-22 Shinkawa-cho, Higashikurume-shi, Tokyo 2030013, Japan. ${ }^{8}$ Kodaira Kitaguchi Clinic, 2-2-11 Onuma-cho, Kodaira-shi, Tokyo 1870001, Japan.

Received: 1 January 2019 Accepted: 2 May 2019

Published online: 30 May 2019

\section{References}

1. Masakane I, Taniguchi M, Nakai S, Tsuchida K, Goto S, Wada A, et al. Annual Dialysis data report 2015, JSDT renal data registry. Renal Replacement Therapy. 2018;4:19 https://doi.org/10.1186/s41100-018-0149-8. Accessed 28 Mar 2018.

2. Kim JC, Kalantar-Zadeh K, Kopple JD. Frailty and protein-energy wasting in elderly patients with end stage kidney disease. J Am Soc Nephrol. 2013;24:337-51.

3. Kim JK, Choi SR, Choi MJ, Kim SG, Lee YK, Noh JW, et al. Prevalence of and factors associated with sarcopenia in elderly patients with end-stage renal disease. Clin Nutr. 2014;33:64-8.

4. Drost D, Kalf A, Vogtlander N, van Munster BC. High prevalence of frailty in end-stage renal disease. Int Urol Nephrol. 2016:48:1357-62.

5. Avin KG, Moorthi RN. Bone is not alone: the effects of skeletal muscle dysfunction in chronic kidney disease. Curr Osteoporos Rep. 2015;13:173-9.

6. Walker SR, Wagner M, Tangri N. Chronic kidney disease, frailty, and unsuccessful aging: a review. J Ren Nutr. 2014;24:364-70.

7. Xue QL. The frailty syndrome: definition and natural history. Clin Geriatr Med. 2011;27:1-15.

8. Beaudart C, Zaaria M, Pasleau F, Reginster JY, Bruyère $O$. Health outcomes of sarcopenia: a systematic review and meta-analysis. PLoS One. 2017;17:e0169548.

9. Onoue $Y$, Izumiya $Y$, Hanatani TT, Yamamura S, Kimura $Y$, et al. A simple sarcopenia screening test predicts future adverse events in patients with heart failure. Int J Cardiol. 2016;215:301-6.

10. Fried LP, Tangen CM, Walston J, Newman AB, Hirsch C, Gottdiener J, et al. Frailty in older adults: evidence for a phenotype. J Gerontol A Biol Sci Med Sci. 2001;56:M146-56.

11. Cesari M, Landi F, Vellas B, Bernabei R, Marzetti E. Sarcopenia and physical frailty: two sides of the same coin. Front Aging Neurosci. 2014;6:192.

12. Sergi G, Veronese $N$, Fontana L, De Rui M, Bolzetta F, Zambon S, et al. Pre-frailty and risk of cardiovascular disease in elderly men and women the ProVA study. J Am Coll Cardiol. 2015;65:976-83.

13. Srikanthan $\mathrm{P}$, Horwich TB, Tseng $\mathrm{CH}$. Relation of muscle mass and fat mass to cardiovascular disease mortality. Am J Cardiol. 2016;117:1355-60.

14. Fonseca Alves DJ, Bartholomeu-Neto J, Júnior ER, Ribeiro Zarricueta BS, Nóbrega OT, Córdova C. Walking speed, risk factors, and cardiovascular events in older adults-systematic review. J Strength Cond Res. 2017;31:3235-44.

15. Wu Y, Wang W, Liu T, Zhang D. Association of Grip Strength with Risk of allcause mortality, cardiovascular diseases, and Cancer in community-dwelling populations:a meta-analysis of prospective cohort studies. J Am Med Dir Assoc. 2017;18:551.e17-35.

16. Andersen K, Rasmussen F, Held C, Neovius M, Tynelius P, Sundström J. Exercise capacity and muscle strength and risk of vascular disease and arrhythmia in 1.1 million young Swedish men:cohort study. BMJ. 2015;351:h4543.

17. Foley RN, Parfrey PS, Sarnak MJ. Clinical epidemiology of cardiovascular disease in chronic renal disease. Am J Kidney Dis. 1998;32(5 Suppl 3):S112-9.

18. Mahoney FI, Barthel DW. Functional evaluation: the Barthel index. Md State Med J. 1965;14:61-5. 
19. Studenski S, Perera S, Wallace D, Chandler JM, Duncan PW, Rooney E, et al. Physical performance measures in the clinical setting. J Am Geriatr Soc. 2003;51:314-22.

20. Charlson ME, Pompei P, Ales KL, MacKenzie CR. A new method of classifying prognostic comorbidity in longitudinal studies: development and validation. J Chronic Dis. 1987:40:373-83.

21. Bonsdorff MBV, Groffen DA, Vidal JS, et al. Rantanen T, Jonsson PV, Garcia M et al. coronary artery calcium and physical performance as determinants of mortality in older age: the AGESReykjavik study. Int J Cardiol. 2013;168:2094-9.

22. Elbaz A, Sabia S, Brunner E, Shipley M, Marmot M, Kivimaki M, et al. Association of walking speed in late midlife with mortality: results from the Whitehall II cohort study. Age (Dordr). 2013;35:943-52.

23. Kuo HK, Yu YH. The relation of peripheral arterial disease to leg force, gait speed, and functional dependence among older adults. J Gerontol A Biol Sci Med Sci. 2008;63:384-90.

24. McDermott MM, Guralnik JM, Ferrucci L, Criqui MH, Greenland P, Tian L, et al. Functional decline in lower-extremity peripheral arterial disease: associations with comorbidity, gender, and race. Vasc Surg. 2005;42:1131-7.

25. McDermott MM, Carroll TJ, Kibbe M, Kramer CM, Liu K, Guralnik JM, et al. Proximal superficial femoral artery occlusion, collateral vessels, and walking performance in peripheral artery disease. JACC Cardiovasc Imaging. 2013;6:687-94

26. Semba RD, Bandinelli S, Sun K, Guralnik JM, Ferrucci L. Relationship of an advanced glycation end product, plasma carboxymethyl-lysine, with slow walking speed in older adults: the InCHIANTI study. Eur J Appl Physiol. 2010;108:191-5.

27. Welmer AK, Angleman S, Rydwik E, Fratiglioni L, Qiu C. Association of cardiovascular burden with mobility limitation among elderly people: a population based study. PLoS One. 2013;8:1-7.

28. Peel NM, Kuys SS, Klein K. Gait speed as a measure in geriatric assessment in clinical settings: a systematic review. J Gerontol A Biol Sci Med Sci. 2013:68:39-46.

29. Cruz-Jentoft AJ, Baeyens JP, Bauer JM, Boirie Y, Cederholm T, Landi F, et al. Sarcopenia: European consensus on definition and diagnosis: report of the European working group on sarcopenia in older people. Age Ageing. 2010;39:412-23.

30. Leong DP, Teo KK, Rangarajan S, Lopez-Jaramillo P, Avezum A Jr, Orlandini A, et al. Prognostic value of grip strength: findings from the prospective urban rural epidemiology (PURE) study. Lancet. 2015;386:266-73.

31. Strand BH, Cooper R, Bergland A, Jørgensen L, Schirmer H, Skirbekk V, et al. The association of grip strength from midlife onwards with all-cause and cause-specific mortality over 17 years of follow-up in the Troms $\varnothing$ study. J Epidemiol Community Health. 2016;70:1214-21.

32. Nofuji $Y$, Shinkai S, Taniguchi $Y$, Amano H, Nishi M, Murayama H, et al. Associations of walking speed, grip strength, and standing balance with Total and cause-specific mortality in a general population of Japanese elders. J Am Med Dir Assoc. 2016:17:184.e1-7.

33. Kishimoto $H$, Hata J, Ninomiya T, Nemeth H, Hirakawa Y, Yosida D, et al. Midlife and late life handgrip strength and risk of cause-specific death in a general Japanese population: the Hisayama study. J Epidemiol Community Health. 2014:68:663-8.

34. Kim JK, Kim SG, Oh JE, Lee YK, Noh JW, et al. Impact of sarcopenia on long-term mortality and cardiovascular events in patients undergoing hemodialysis. Korean J Intern Med. 2019;34:599-607.

35. Roberts HC, Denison HJ, Martin HJ, Patel HP, Syddall H, Cooper C, et al. A review of the measurement of grip strength in clinical and epidemiological studies: towards a standardised approach. Age Ageing. 2011:40:423-9.

36. Kong YK. The effects of co-ordinating postures with shoulder and elbow flexion angles on maximum grip strength and upper-limb muscle activity in standing and sitting postures. Int J Occup Saf Ergon. 2014;20:595-606.

37. Liao WC, Wang CH, Yu SY, Chen LY, Wang CY. Grip strength measurement in older adults in Taiwan: a comparison of three testing positions. Australas J Ageing. 2014;33:278-82.

38. Rehfuss JP, Berceli SA, Y BSMH, Kubilis PS, Beck AW, Huber TS, et al. The spectrum of hand dysfunction after hemodialysis fistula placement. Kidney Int Rep. 2017;2:332-41.
39. Kmentova T, Valerianova A, Kovarova L, Lachmanova J, Hladinova Z, Malik J. Decrease of muscle strength in vascular access hand due to silent ischaemia. J Vasc Access. 2018;1:1129729818763287.

40. Saevereid HA, Schnohr P, Prescott E. Speed and duration of walking and other leisure time physical activity and the risk of heart failure: a prospective cohort study from the Copenhagen City heart study. PLoS One. 2014;9:e89909.

41. Stamatakis E, Kelly P, Strain T, Murtagh EM, Ding D, Murphy M. Self-rated walking pace and all-cause, cardiovascular disease and cancer mortality: individual participant pooled analysis of 50225 walkers from 11 population British cohorts. Br J Sports Med. 2018:52:761-8.

42. Artero EG, Lee DC, Lavie CJ, España-Romero V, Sui X, Church TS, et al. Effects of muscular strength on cardiovascular risk factors and prognosis. J Cardiopulm Rehabil Prev. 2012;32:351358.

43. Fritz NE, McCarthy CJ, Adamo DE. Handgrip strength as a means of monitoring progression of cognitive decline - a scoping review. Ageing Res Rev. 2017;35:112-23.

44. Lee SY, Yang DH, Hwang E, Kang SH, Park SH, Kim TW, et al. The prevalence, association, and clinical outcomes of frailty in maintenance Dialysis patients. J Ren Nutr. 2017:27:106-12.

\section{Ready to submit your research? Choose BMC and benefit from:}

- fast, convenient online submission

- thorough peer review by experienced researchers in your field

- rapid publication on acceptance

- support for research data, including large and complex data types

- gold Open Access which fosters wider collaboration and increased citations

- maximum visibility for your research: over $100 \mathrm{M}$ website views per year

At BMC, research is always in progress.

Learn more biomedcentral.com/submissions 